\title{
Passage, Transition, Transformation? Die Sozialen Passagen als Ort sozialpädagogischer Reflexion
}

\section{Ein Essay anlässlich des zehnjährigen Bestehens der Zeitschrift Soziale Passagen: Journal für Empirie und Theorie Sozialer Arbeit}

\section{Johanna Mierendorff}

Zusammenfassung Anlässlich des zehnjährigen Jubiläums des Journals ,,Soziale Passagen" setzt sich der Beitrag mit der Gründungsgeschichte und der inhaltlichen Ausrichtung der Zeitschrift auseinander. Mit der Gründung der Zeitschrift ist ein neuer Ort sozialpädagogischer Reflexion entstanden, an dem sozialpädagogisches Handeln und sozialpädagogische Problemfelder unter den Bedingungen umfassender gesellschaftlicher Transformation empirisch und theoretisch in den Blick genommen werden. Das Journal Soziale Passagen kann damit den Anspruch geltend machen, Teil des Professionalisierungsprozesses der Sozialen Arbeit zu sein.

Schlüsselwörter Statuspassagen · Transition · Transformation ·

Professionalisierung · Reflexive Sozialpädagogik

Dieser Beitrag basiert auf dem Festvortrag, der im Rahmen der Veranstaltung anlässlich des zehnjährigen Jubiläums der Zeitschrift Soziale Passagen: Journal für Empirie und Theorie Sozialer Arbeit am 6. Juli 2019 in der Tagungsstätte Haus Neuland, Bielefeld, gehalten wurde.

Prof. Dr. J. Mierendorff $(\bowtie)$

Institut für Pädagogik, Philosophische Fakultät III, Martin-Luther-Universität Halle-Wittenberg,

Franckeplatz 1, 06099 Halle, Deutschland

E-Mail: johanna.mierendorff@paedagogik.uni-halle.de 


\title{
Status passages, transition, transformation? The journal Soziale Passagen as a place of professional reflection
}

An essay on the occasion of the 10th anniversary of the magazine Soziale Passagen: Journal für Empirie und Theorie Sozialer Arbeit

\begin{abstract}
The scientific essay, written in behalf of the decennial of the magazine, focuses on the founding history and the topic of Soziale Passagen. It argues that a new academic place of professional reflection has been established. Starting point was the identification of an enormous process of societal transitions which strongly influences social work. The journal is identified as an important player in the process of professionalization.
\end{abstract}

Keywords Transition · Transformation · Professionalization

Das zehnjährige Jubiläum der Zeitschrift Soziale Passagen bietet den Anlass zurückzuschauen und eine Auseinandersetzung mit den Motiven ihrer Gründung, der Begründung ihres Gegenstandes sowie deren Bedeutung für die Weiterentwicklung einer reflexiven Profession zu führen. Der Blick in das Editorial der ersten Ausgabe gibt Aufschluss über deren Grundausrichtung. Erstens ist mit dem Titel der Zeitschrift Soziale Passagen der Fokus deutlich gesetzt: Es geht um gesellschaftliche, institutionelle und biographische Passagen - um Transitionen und Transformationen, wenn man den Begriff der Passage weit auslegt. Zweitens sollen bei der Auseinandersetzung mit diesem Themenfeld sowohl soziologisch-sozialwissenschaftliche, erziehungswissenschaftliche und sozialpädagogische Perspektiven eingenommen werden - diese Perspektivierungen sind, betrachtet man die Ausgaben bis heute, Ausdruck der programmatisch-interdisziplinären Ausrichtung. Es zeigen sich der Wille der Herausgeberinnen und Herausgeber zu disziplinärer Grenzüberschreitung im Denken und damit möglicherweise das Unscharf-Werden von Disziplingrenzen. Dennoch versteht sich die Zeitschrift nicht als interdisziplinäre, sondern explizit - das ist deutlich formuliert - als disziplinär im Sozialpädagogischen verhaftetes Journal (vgl. Bock et al. 2009a).

Im Folgenden werde ich sowohl die interdisziplinäre, disziplinüberschreitende Ausrichtung wie den Gegenstand der Zeitschrift - Passagen, Transitionen, Transformationen - würdigen. Hierauf aufbauend werde ich nach der Bedeutung des Journals für die Profession in Zeiten fortschreitender sozialer Transformationen und damit nach der Bedeutung dieser Ausrichtung für den Reflexivitätsanspruch der Sozialen Arbeit als Profession fragen. Annähern werde ich mich in einer suchenden Bewegung. Das Format eines wissenschaftlichen Essays erscheint hierfür geeignet, denn es liegt dem Beitrag ausschließlich die Lektüre der Hefte, aber keine umfassende wissenschaftliche Analyse zugrunde. 


\section{1 Über das Heft, seine Herausgeber*innen und deren Beobachtung gesellschaftlicher Transformation}

Was mag die Gründungsherausgeberinnen und -herausgeber Karin Bock, Karin Böllert, Werner Thole und Holger Ziegler Mitte der 2000er Jahre dazu bewogen haben, eine neue Zeitschrift zu gründen? Zeitschriften der Sozialen Arbeit und der Sozialpädagogik - praxisnahe aber auch theoretisch ausgerichtete - gab es zu diesem Zeitpunkt bereits eine große Zahl. Die Deutsche Gesellschaft für Soziale Arbeit (DGSA) hat in ihrer Sammlung: „Fachzeitschriften der Sozialen Arbeit und ihrer Bezugswissenschaften“"vom 13. Dezember 2018 insgesamt 178 Zeitschriften zusammengestellt, die für die Soziale Arbeit relevant seien. Dennoch schien der Markt entweder noch nicht gesättigt oder aber die Ausrichtung bisheriger Zeitschriften als ungenügend. Ich gehe eher von Letzterem aus. Warum aber das? Was eint die Herausgeberinnen und Herausgeber, was bringt sie dazu, ein neues Format auf den Markt und in die Disziplin zu werfen? Interessant erscheint mir, dass die Forschungsschwerpunkte oder besser die systematischen und thematischen Ausrichtungen der Gründungsmütter und Gründungsväter in den Bereichen Wohlfahrtsstaat, Sozialpolitik sowie sozialer und gesellschaftlicher Wandel liegen und diese Orientierung sowohl in der empirischen Forschung, in der Theoriebildung wie auch in deren (fach)politischem Engagement zu finden ist. Die Herausgeberinnen und Herausgeber fokussieren in ihren Forschungsarbeiten nicht allein Mikropraktiken und Methoden der Sozialen Arbeit oder aber Institutionen- oder Adressat*innenproblematiken, sondern die komplexen Zusammenhänge gesellschaftlicher Verfasstheit, sozialpolitischer Gestaltung des Sozialen und der Sozialen Arbeit als eine der Voraussetzungen moderner, kapitalistischer, gewinnorientierter, kompetitiver Ökonomien. Das ist der Denkhorizont der Gründungsmitglieder, wenngleich nicht die alleinige Ausrichtung der konkreten Forschungsarbeiten.

Deutlich wird nun in den ersten Ausgaben der Sozialen Passagen, dass die wie auch immer motivational Verbundenen nicht allein jenen interdisziplinären Denkhorizont teilen, sondern vor allem die Beobachtung einer entgrenzten Moderne und des fortschreitenden sozialen und wohlfahrtsstaatlichen Wandels (vgl. Bock et al. 2009a). Unterstellt wird, dass damit auch die Bedingungen der Sozialen Arbeit, die als wohlfahrtsstaatlich verfasst angesehen werden, im Wandel sind und sich daher Disziplin und Profession konsequenterweise verändern. Es erscheint plausibel, anzunehmen, dass die gemeinsame Beobachtung einer umfassenden Transformation diese Gruppe wohlfahrtsstaatstheoretisch orientierter Forscherinnen und Forscher veranlasst hat, eine „Publikationsplattform“ (Bock et al. 2009a, S. 4) zu schaffen, auf der diese Feststellung theoretisch und empirisch zum Gegenstand der (inter)disziplinären Auseinandersetzung wird. Damit ist ein Ort der wissenschaftlichen Reflexion des sozialpädagogischen Tuns, der Institutionen und Adressat*innen der Sozialen Arbeit geschaffen worden, an dem der aktuelle rapide Wandel explizit sowohl zum Ausgangspunkt als auch zum Gegenstand gemacht wurde und damit nicht nur in einer Zeit des diskursiven Hypes kurzzeitig neben anderen Themen aufgegriffen wird. Vielmehr ist eine dauerhafte und systematische Auseinandersetzung mit langfristigen und umfassenden Wandlungsprozessen angestrebt gewesen. Das grenzt die Sozialen Passagen deutlich von anderen Fachzeitschriften ab. Ziel war es aber auch, 
„Soziale Arbeit wissenschaftlich weiter [zu] konturieren“ (Bock et al. 2009b, S. 129) und so deren Professionalisierungsanliegen gerade auch in Zeiten des Wandels voranzutreiben. Denn Professionalisierung ohne theoretische Reflexion erscheint nicht denkbar (vgl. Müller 2012; Dollinger 2008). Der Gegenstand Transformation ist ein wichtiger Gegenstand eines solchen Reflexionsprozesses, sind Transformationen, Transitionen und Passagen doch unabhängig vom aktuellen gesellschaftlichen Wandel generell zentrale Bezugspunkte sozialpädagogischen Handelns (s. unten).

Die Feststellung der tiefgreifenden Veränderungen in der Struktur der Arbeitswelt, der Kultur und des Sozialen dieser Gesellschaft und den damit verbundenen sozialen Folgen sowie die Rolle der Sozialen Arbeit in diesem Prozess der Neujustierung des Sozialen steht im Mittelpunkt der Zeitschrift und bildet sich in den Themenheften bis heute ab. Disziplinäre Perspektivwechsel, interdisziplinäre Perspektiven galten und gelten als Voraussetzung, um diese umfassenden Transformationsprozesse erfassen zu können - so ist es im Editorial der ersten beiden Themenhefte formuliert. Diesem Gedanken möchte ich einen historisch rückwärtsgewandten hinzufügen. Interdisziplinarität, Grenzgängertum, Transformationen, Passagen sind Teil der Gründungsgeschichte der Sozialen Arbeit. Erinnert sei an die Wurzeln der Sozialen Arbeit in den USA - die Chicago School, das Werk „The polish peasant“, die Settlement Hull House Bewegung. Dayana Lau (2019) hat in ihrer disziplingeschichtlich wichtigen Dissertationsschrift mit dem Titel „Frühe Forschungspraxis und die Differenzierung von Disziplin und Profession in der Sozialen Arbeit in den USA. Zum Verhältnis von Sozialreform und Wissenschaft (1895-1920)“ die in den USA zunächst kaum vorhandene disziplinäre Trennung von Sozialer Arbeit und Soziologie herausgearbeitet. Sie rekonstruiert die spannungsgeladene Ausdifferenzierung der Sozialen Arbeit als Profession und der Soziologie als wissenschaftliche Disziplin in den Jahren ihrer Etablierung. In den Blick genommen wird die frühe Chicagoer Forschungstradition, die sich zunächst in enger Kooperation zwischen dem Settlement Hull House und dem Institut für Soziologie herausgebildet hat. Dayana Lau (2019) zeigt auf, dass die konkrete Ausformung der Sozialen Arbeit als professionelle Handlungspraxis auch als eine Folge der Anstrengungen der Soziologie, sich als universitäre Disziplin zu etablieren, interpretiert werden kann. Interdisziplinarität, Abgrenzungen und die erneute Bewegung aufeinander zu sind Teil der Geschichte der Sozialen Arbeit. In Westdeutschland kann ein Changieren der Sozialpädagogik zwischen Soziologie und Erziehungswissenschaft beobachtet werden. Dies zeigt sich bspw. daran, dass die seit 1969 eingerichteten sozialpädagogischen Studiengänge an westdeutschen Universitäten (Rauschenbach 1991, S. 5) zum Teil in erziehungswissenschaftlichen Einheiten, zum Teil aber auch an sozial- bzw. gesellschaftswissenschaftlichen Fachbereichen implementiert wurden, wie bspw. an den Universitäten in Duisburg und Wuppertal. Gleichermaßen verortete sich die Sozialpädagogik aber disziplinär in der Deutschen Gesellschaft für Erziehungswissenschaften, nicht aber in der Deutschen Gesellschaft für Soziologie. Ein weiteres Beispiel für dieses Changieren: In der 1949 gegründeten soziologischen Zeitschrift „Soziale Welt: Zeitschrift für sozialwissenschaftliche Forschung“ - damals führte sie noch den Titel „Soziale Welt - eine Zeitschrift für Wissenschaft der Praxis des sozialen Lebens" - wurden bis Mitte der 1960er Jahre explizit auch sozialpädagogische Themen verhandelt. Der Schwerpunkt der Zeitschrift lag in Fragen der industriellen Arbeitswelt und Sozial- 
politik. Seit Ende der 1960er Jahre verschob sich der Fokus dann aber auf ,spezielle Themen soziologischer Theorie, auf die Methodik der empirischen Sozialforschung und auf Probleme gesellschaftlicher Organisationen“" (Hartmann 1979, S. 1) - mit dieser strengeren soziologischen, auch grundlagentheoretischen Fokussierung waren sozialpädagogische Themen anscheinend nicht mehr von Interesse und damit die „Soziale Welt“" kein Publikationsort mehr für die Sozialpädagogik.

Ein weiterer Gedankengang ist inspiriert durch die Lektüre der ersten Heftausgaben und den dort formulierten Legitimationen für eine neue Zeitschrift: Im Editorial des ersten Heftes ist moniert, dass sich in dem historischen Prozess gesellschaftlicher Arbeitsteilung und Ausdifferenzierung eine ,Zerfaserung der empirischen und theoretischen Grundlagen der Sozialen Arbeit“" (Bock et al. 2009a) vollzogen habe ${ }^{1}$. Das beschäftigt die Herausgeberinnen und Herausgeber: Ein Kontrapunkt solle gesetzt, ein Ort der sozialpädagogisch-theoretischen Fokussierung geschaffen werden, der die Ausdifferenziertheit nicht negiere, aber einen gemeinsamen Fluchtpunkt biete. Fluchtpunkt sind Theorien und Empirien der Sozialen Arbeit. Geschaffen werden solle über handlungsfeldbezogene Grenzen hinweg ein Ort der disziplinären Verhandlung von Theorien und Methoden Sozialer Arbeit unabhängig von ihrer handlungsfeldbezogenen Ausdifferenziertheit. Geht es also, wie Michael Winkler (2003) problematisiert hat, in dieser Zeitschrift um eine Besinnung auf den schwer beobachtbaren und schwer bestimmbaren Kern der Sozialen Arbeit? Vielleicht, oder doch wohl eher nicht. Ausgehend von der Feststellung und theoretisch-empirischen Bearbeitung des sozialen und damit auch des fachlichen und disziplinären Wandels ist das Ziel eher eine theoretische Fokussierung veränderter Bedingungen sozialpädagogischen Handels in der Breite, die die handlungsfeldbezogene Ausdifferenzierung im Denken überschreitet, aber nicht negiert. Die Bewegungen der Zeitschrift sind also zugleich interdisziplinär öffnend und durch sozialpädagogische Fokussierungen wieder schließend.

\section{Passagen, Transitionen, Transformationen - Gedankenspiele zum Gegenstand der Zeitschrift}

Passagen, Transitionen und Transformationen sind beständiger Teil unseres alltäglichen, professionellen und auch wissenschaftlichen Denkens. Unsere Bibliotheken und Aktenschränke im öffentlichen und privaten Raum zeugen davon: So berichtet die biblische Geschichte von der Transformation des Saulus zum Paulus oder aber über den Wandel der Sünderin Maria von Magdala zu einer Jüngerin Jesu. Auch die schöne Literatur ist voll von poetischen Passagenbeschreibungen - Johann Wolfgang Goethes wichtiger Bildungsroman „Wilhelm Meisters Lehrjahre“, Thomas Manns „Joseph und seine Brüder“, Doris Dörries spöttelnder Roman „Beim nächsten Mann wird alles anders“ - in unseren Bücherschränken ließen sich unzählige weitere Beispiele dafür finden. Voll von Passagen- und Transitionsbeschreibungen sind aber

\footnotetext{
${ }^{1}$ Deutlich wird in der bereits genannten Sammlung „Fachzeitschriften der Sozialen Arbeit und ihrer Bezugswissenschaften“ der DGSA, dass für jede Teildisziplin, jedes Handlungsfeld, jede Lebenslage ein eigener Publikationsort etabliert wurde.
} 
auch die Aktenschränke der Sozial- und Jugendämter oder der Wohlfahrtsorganisationen: dokumentieren sie doch gelungene und nicht gelungene Statuspassagen und Transitionen von Individuen und Gruppen. Ziel Sozialer Arbeit ist es unter anderem, die Adressat*inne dabei zu unterstützen, ein Leben ohne professionelle sozialpädagogische Unterstützung oder Begleitung zu führen. Diese Prozesse werden dokumentiert - muss doch die Fortsetzung oder das Ende einer Hilfe begründet sein und legitimiert werden. Erst die dokumentierte gelungene Transition oder aber die Unmöglichkeit der Transition begründen bspw. das Ende einer Hilfe.

Auch die Sozialwissenschaften beschäftigen sich seit Mitte der 1980er Jahre in ihren institutionalisierten Zusammenschlüssen explizit mit den Begriffen der Passage, der Transition oder der Transformation. Zu nennen wäre der Bremer Sonderforschungsbereich 186 mit dem Titel „Statuspassagen und Risikolagen im Lebensverlauf: institutionelle Steuerung und individuelle Handlungsstrategien" (1988-2001)2, der Kongress der Deutschen Gesellschaft für Soziologie im Jahr 2008 in Jena zum Thema „Unsichere Zeiten. Herausforderungen gesellschaftlicher Transformationen “ oder aber das Graduiertenkolleg „Widersprüche gesellschaftlicher Transformation. Soziale Arbeit in der Transformation“" (2013-2016, Düsseldorf, Köln, Duisburg-Essen).

Nicht erst der genannte Sonderforschungsbereich macht darauf aufmerksam, dass Statuspassagen im Lebenslauf als risikoreich eingeschätzt werden. Der moderne Lebenslauf sei von seiner Grundstruktur her, folgt man Martin Kohli (1985), dreigeteilt. Er berge zwei große biographische Statuspassagen in sich: Eine erste Statuspassage im Sinne dieser Einteilung ist der Austritt aus dem Bildungsmoratorium Kindheit und der Eintritt in die Phase des erwerbsarbeitszentrierten Erwachsenseins. An diesem Übergang bestehe das Risiko des schulischen Scheiterns und damit das Risiko des Scheiterns einer normativ anstrebenswerten ungebrochenen Erwerbsbiographie. Oder auch - das hat die Familiensoziologie gezeigt - das drohende Scheitern des Eingehens einer Partnerschaft zum Zwecke der Gründung einer Familie. Zweite Statuspassage im Kohlischen Sinne ist der Übergang von der aktiven Erwerbsphase in den Ruhestand. Zu bewältigen ist das psychisch risikoreiche und ökonomisch vielfach problematische Ausscheiden aus dem Beruf. $\mathrm{Zu}$ bearbeiten ist gegebenenfalls auch der für Mütter und Väter und für die Partnerschaft teilweise krisenhafte Übergang von der aktiven Phase der Kinderziehung in die Empty-Nest-Phase. Martin Kohli und Familiensoziolog*innen gehen hier normativ von erwerbsarbeits- und familienzentrierten Normalbiographien aus. Auch wenn spätestens seit Ulrichs Becks (1986) Feststellung der „Risikogesellschaft“ solche Normalverläufe nicht mehr unhinterfragt angenommen werden können, bleibt der Kern dieser Systematik in spezifischer Weise plausibel. Das System der sozialen Sicherung ist genau um diese beiden Risikopassagen herum aufgebaut, mit dem Ziel, diese abzusichern und Problemverläufe zu verhindern (Bildungs-, Erziehungs- und Sozialsystem mit seinen passagenunterstützenden flankierenden Angeboten, Arbeitslosensicherung, Rentenversicherung). In einer flexiblen, hochgradig ausdifferenzierten und entgrenzten Risikogesellschaft lassen sich darin und drum herum weitere risikoreiche biographische Passagen und Transitionen nennen: Schulwechsel, Wechsel der Erwerbstätigkeit oder

\footnotetext{
${ }^{2}$ Im Abschlussbericht heißt es: „Es geht um die Bewältigung diskontinuierlicher Statuspassagen.“
} 
Verlust der Erwerbsarbeit, Wechsel oder Verlust eines Partners, Umzüge aufgrund von Arbeitslosigkeit, Heirat, Scheidung, Wiederheirat etc.

Alle diese Passagen werden in ihrem Transitionsgeschehen als potentiell vom Scheitern bedroht betrachtet. Der Ausgang dieser Prozesse ist stets ungewiss, der Verlauf möglicherweise gefährlich für das Individuum und im Falle eines massenhaften Auftretens auch für die soziale Ordnung. Zu erinnern ist an die historische Diskussion um die gefährliche Lücke zwischen Schule und Kasernentor und die als gefährdet bzw. gefährlich angenommene männliche Jugend zu Beginn des 20. Jahrhunderts (vgl. Sachße und Tennstedt 1988) oder aber an die Sorge einer ganzen Reihe von Familiensoziolog*innen Ende der 1980er Jahre über die Zunahme von nichtehelichen Lebensgemeinschaften und die damals angenommene Gefährdung der Sozialisationsfähigkeit der modernen Familie (vgl. u. a. Herlth et al. 1994).

Als besonders prekär und risikoreich gelten biographische Transitionsprozesse in Zeiten gesellschaftlicher und politischer Transformation und Instabilität. Deutlich wurde dies bspw. im Prozess der sogenannten deutsch-deutschen Wiedervereinigung nach 1989. Die „Kommission zur Erforschung des sozialen und politischen Wandels in den neuen Bundesländern in der Bundesrepublik Deutschland“ hat im Rahmen ihrer umfassenden Forschungen in den Sektoren Politik, Arbeit, Sozialstruktur und soziale Sicherheit den ostdeutschen Transformationsprozess untersucht und die ungeheuren Risiken, Umbrüche und Verwerfungen für den einzelnen und für das Soziale aufgedeckt (vgl. Bertram und Kollmorgen 2001). Die Folgen der Erschütterung des Sozialen sehen wir bis heute.

Instrumente der institutionalisierten und professionellen Bearbeitung solcher Passagen sind unterschiedliche, auch sozialpädagogische Programme, die an dem Risiko des oder dem sich vollziehenden Scheitern ansetzen. Exemplarisch genannt werden können die Frühen Hilfen, die verhindern sollen, dass sich nach der Geburt eines Kindes ein gefährlicher Sozialisations- und Erziehungsprozess in den Familien vollziehe, da Eltern überfordert oder inkompetent seien. Zu nennen wären auch die in Kindertagesstätten seit Anfang der 2000er Jahre etablierten Programme zum „Übergang vom Kindergarten in die Schule“, die zum Ziel haben, den risikoreichen Transitionsprozess des Kindes zu unterstützen und durch einen bruchlosen Übergang einem Scheitern der Bildungskarriere vorzubeugen. Erwähnt werden können weiterhin die Angebote der Berufsberatung und vor allem das Berufsgrundbildungsjahr (BGJ) und das Berufsvorbereitungsjahr (BVJ) für Jugendliche ohne Schulabschluss, die Angebote der Familienbildungsstätten für werdende Eltern, die soziale Altenbildungsarbeit für die Neu-Senior*in, die Fallmanager*in auf dem Arbeitsamt, die eigentlich nicht nur verwalten, sondern die Passage zurück in den Arbeitsmarkt beratend begleiten sollte. Viele der sozialpädagogischen Angebote setzen in biographischen Passagen ein.

Man kann also davon sprechen, dass Soziale Arbeit Statuspassagen und Transitionen auf zweierlei Weisen bearbeitet:

- Präventiv in der Annahme der generellen Risikobehaftetheit eines biographischen Übergangs (bspw. das Elternwerden, der Eintritt in die oder der Austritt aus der Schule oder Erwerbsarbeit, das Rentner*inwerden. Hier finden wir bspw. Beratungen, Schulungen, Trainings). 
- Eingreifend, wenn Übergänge prekär zu werden drohen, prekär geworden sind oder scheitern (bspw. Sozialpädagogische Familienhilfe, Frühe Hilfen, Berufsgrundbildungsjahr).

Zugrunde liegen diesen Programmen, Maßnahmen und Hilfen normative Annahmen über ein gelingendes Leben und nach wie vor über Normalbiographien - Martina Richter (2013) hat dies bspw. am Beispiel der Sozialpädagogischen Familienhilfe gezeigt. Präventive und eingreifende Maßnahmen setzen auf unterschiedlichen Ebenen an, nehmen unterschiedliche Problemkonstellationen in den Blick: Passagenprobleme auf personaler oder familialer Ebene, auf der Ebene nicht-familialer Gruppen sowie Transformationen, die sich im Raum vollziehen (z. B. in Quartieren oder administrativ bestimmten Sozialräumen, die zum „sozialen Brennpunkt“ zu werden drohen).

Was ist also Soziale Arbeit in Bezug auf individuelle Status- und Risikopassagen und im Kontext eines beobachtbaren umfassenden gesellschaftlichen Transformationsgeschehens? Soziale Arbeit ist Passagenbegleiterin, Passagenermöglicherin, Passagenkontrolleurin. Passagen - von einer Lebenslage in die andere oder von einer Lebensphase in die andere - können durch sozialpädagogisch ausgebildete Fachkräfte begleitet werden. Im Verlauf umfassender gesellschaftlicher Transformationsprozesse ist Soziale Arbeit in diesen Rollen besonders gefragt, da die an sich risikobehafteten Statuspassagen besonders gefährlich erscheinen oder sind. Soziale Arbeit ist also ein Ort der Bewältigung von Statuspassagen. Soziale Arbeit ist eine Akteurin im Prozess der Bearbeitung von Statuspassagen, Transitionen und Transformationen.

Abschließend sei ein weiterer Gedanke angeschnitten. Die Institutionen der Sozialen Arbeit, die Profession und die Disziplin, machen selbst Transformationsprozesse durch ${ }^{3}$. Zu nennen wäre bspw. die Durchsetzung neuer Steuerungsmodelle seit den 1980er Jahren in der Sozialen Arbeit, insbesondere auch in der Kinder- und Jugendhilfe - diese haben einen bedeutenden, intendierten Transformationsprozess der Organisation der Sozialen Arbeit ausgelöst. Das Eindringen kompetitiver, betriebswirtschaftlicher Grundprinzipien in die Steuerung hat erhebliche Konsequenzen für die Adressat*innen, das Verhältnis zwischen Adressat*innen und Professionellen, für die Arbeits- und Verwaltungsprozesse, für die tägliche Organisation der Sozialen Arbeit. Zu nennen wären darüber hinaus aber auch Transformationen, die sich aufgrund von sich verändernden Lebenslagen der Adressat*innen oder aufgrund von veränderten sozialen Problemkonstellationen vollziehen und neue Anforderungen an die Handlungsfelder der Sozialen Arbeit mit sich bringen. In allen genannten Beispielen ist Soziale Arbeit gezwungen, sich ebenfalls zu transformieren, ihre Instrumente ggf. anzupassen, neue Handlungsfelder zu erschließen.

\footnotetext{
${ }^{3}$ In diesem Kontext der Institution ist kaum von Passagen zu sprechen, da vom Grundsatz her erst einmal kein Entwicklungsprozess auf ein bestimmtes Ziel hin vorausgesetzt und angestrebt ist - wie beim Menschen vom unmündigen Kind zum mündigen Erwachsenen.
} 


\section{Schlussbetrachtungen}

Soziale Arbeit bearbeitet gesellschaftliche Transformationsprozesse in ihren Folgen für das Individuum, für Gruppen und für das Gemeinwesen. Sie unterstützt die Bewältigung von risikoreichen Statuspassagen und Transitionen. Inzwischen beobachtet, reflektiert und korrigiert sie das darauf bezogene eigene Tun fortlaufend und professionell. Solche Reflexionen sind auf unterschiedliche Ebenen bezogen: den gesellschaftlichen Wandel, individuelle Transitionen und Statuspassen, das sozialpädagogische Handeln, die Organisationsformen der Sozialen Arbeit oder aber auf die Transformation der eigenen Profession. Reflexion und Reflexivität sind Teil der Professionalisierung der Sozialen Arbeit. Bernd Dollinger (2008), Reinhardt Hörster (2011) oder Burghardt Müller (2012) haben unterschiedlichste professionalisierungstheoretische Denkangebote in Bezug auf die Reflexionsanforderungen und -prozesse der Sozialen Arbeit vorgeschlagen, die sich sowohl auf das konkrete professionelle Handeln wie auch auf das Verstehen der Professionsgeschichte beziehen. Bernd Dollinger spricht von einer reflexiven Sozialen Arbeit (2008). Die empirische und theoretische Reflexion der Gegenstände Transformation, Transition und biographisch risikoreiche Passage ist das dezidierte Ziel des Journals Soziale Passagen. Indem sie solchen Reflexionen von Transformationsprozessen eine Plattform bietet, ist sie Teil eines die Notwendigkeit der Reflexion anerkennenden Professionalisierungsprozesses. Denn reflektiert wurden in den vergangenen zehn Jahren nicht allein einzelne sozialpädagogische Handlungspraxen, sondern vor allem auch die strukturellen Veränderungen der Institutionen sowie die Folgen veränderter politischer Steuerung für Professionelle, Adressat*innen oder den Organisationsalltag. Solche Reflexionen sind ein wichtiger Bestandteil der Weiterentwicklung einer reflexiven Profession - die Zeitschrift Soziale Passagen ist hierbei ein Instrument, ein Medium. Sie bietet einen Ort der Reflexion.

Die Gründung eines neuen Journals in Zeiten der Entgrenzung scheint also historisch nicht zufällig, sondern als konsequente Folge einer in der Transformation befindlichen Gesellschaft und den damit einhergehenden Folgen für eines diese Prozesse bearbeitenden Teilsystems, nämlich das der Sozialen Arbeit. Weder die herkömmlichen Forschungsgegenstände innerhalb der Disziplingrenzen noch die herkömmlichen Zeitschriften schienen vermutlich hierfür eine befriedigende Grundlage zu bieten. Denn wenn Soziale Arbeit stets Teil gesellschaftlicher Transformationsprozesse ist, sich darin wandelt und transformiert, muss es auch spezifische, darauf ausgerichtete Orte der Reflexion dessen geben. Die Sozialen Passagen sind in diesem Sinne also nicht nur eine Zeitschrift mehr auf dem Markt, sondern ein Format mit einem anderen, querliegenden Zuschnitt in einer Zeit des Brüchig-Werdens bisheriger Normalitätsannahmen.

Abschließend bleibt nur noch zu sagen, dass das bis hierher Festgestellte ein seiner Genese geschuldeter Wissenschaftsessay ist und bleibt, der in der Ausarbeitung vor allem an der Unschärfe in der Bestimmung und der Verwendung der Begriffe Passage, Transition und Transformation leidet. Es bleibt für die Zeitschrift interessant, weitere Selbstreflexionen anzustrengen, vielleicht in ferner Zukunft detailliertere wissenschaftsgeschichtliche Forschungen anzustrengen. Weiterhin erscheint die Frage nach der Rolle einer solchen Zeitschrift in dem nicht abgeschlossenen 
Transformationsprozesses der Gesellschaft, der Ökonomie, des Wohlfahrtsstaates und der damit verbundenen Konsequenzen für Individuen, Institutionen, die Profession und sozialpädagogisches Handeln interessant. Ob es gelingt, das mit der Gründung der neuen Zeitschrift versprochene Nicht-Festhalten am Status Quo weiterhin zu realisieren, ist zu verfolgen. Die Zeitschrift hatte sich zum Ziel gesetzt, ein Ort der Bearbeitung und Analyse gesellschaftlicher Transformationsprozesse in ihren Folgen für die Soziale Arbeit zu untersuchen - langfristig wird sie sich an diesem Ziel und an diesem Anspruch messen lassen müssen.

Funding Open Access funding provided by Projekt DEAL.

Open Access Dieser Artikel wird unter der Creative Commons Namensnennung 4.0 International Lizenz veröffentlicht, welche die Nutzung, Vervielfältigung, Bearbeitung, Verbreitung und Wiedergabe in jeglichem Medium und Format erlaubt, sofern Sie den/die ursprünglichen Autor(en) und die Quelle ordnungsgemäß nennen, einen Link zur Creative Commons Lizenz beifügen und angeben, ob Änderungen vorgenommen wurden.

Die in diesem Artikel enthaltenen Bilder und sonstiges Drittmaterial unterliegen ebenfalls der genannten Creative Commons Lizenz, sofern sich aus der Abbildungslegende nichts anderes ergibt. Sofern das betreffende Material nicht unter der genannten Creative Commons Lizenz steht und die betreffende Handlung nicht nach gesetzlichen Vorschriften erlaubt ist, ist für die oben aufgeführten Weiterverwendungen des Materials die Einwilligung des jeweiligen Rechteinhabers einzuholen.

Weitere Details zur Lizenz entnehmen Sie bitte der Lizenzinformation auf http://creativecommons.org/ licenses/by/4.0/deed.de.

\section{Literatur}

Beck, U. (1986). Risikogesellschaft. Auf dem Weg in eine andere Moderne. Frankfurt am Main: Suhrkamp. Bertram, H., \& Kollmorgen, R. (Hrsg.). (2001). Die Transformation Ostdeutschlands. Berichte zum sozialen und politischen Wandel in den neuen Bundesländern. Opladen: Leske + Budrich.

Bock, K., Böllert, K., Thole, W., \& Ziegler, H. (2009a). Editorial. Soziale Passagen - Journal Für Theorie und Empirie Sozialer Arbeit, 1(1), 3-7.

Bock, K., Böllert, K., Thole, W., \& Ziegler, H. (2009b). Editorial. Soziale Passagen - Journal Für Theorie und Empirie Sozialer Arbeit, 1(2), 129-131.

Dollinger, B. (2008). Reflexive Sozialpädagogik. Struktur und Wandel sozialpädagogischen Wissens. Wiesbaden: VS.

Hartmann, H. (1979). Zum Eintritt in den 30. Jahrgang: Bilanz und Ausblick für die Zeitschrift „Soziale Welt“. Soziale Welt, 30(1), 1-8.

Herlth, A., Brunner, E. J., Tyrell, H., \& Kriz, J. (Hrsg.). (1994). Abschied von der Normalfamilie? Partnerschaft kontra Elternschaft. Berlin: Springer.

Hörster, R. (2011). Sozialpädagogische Kasuistik. In H.-U. Otto \& H. Thiersch (Hrsg.), Handbuch Soziale Arbeit (4. Aufl. S. 1476-1484). München und Basel: Ernst Reinhardt.

Kohli, M. (1985). Die Institutionalisierung des Lebenslaufs: Historische Befunde und theoretische Argumente. Kölner Zeitschrift für Soziologie und Sozialpsychologie, 37, 1-9.

Lau, D. (2019). Soziale Bewegungen, Professionalisierung und Disziplinbildung in der frühen Sozialen Arbeit. Trier: Universität Trier. https://doi.org/10.25353/ubtr-xxxx-0b8a-1687.

Müller, B. (2012). Sozialpädagogisches Können (7. Aufl.). Freiburg im Breisgau: Lambertus.

Rauschenbach, T. (1991). Sozialpädagogik - eine akademische Disziplin ohne Vorbild? neue praxis, 21(1), $1-11$.

Richter, M. (2013). Die Sichtbarmachung des Familialen. Gesprächspraktiken in der Sozialpädagogischen Familienhilfe. Weinheim und Basel: Beltz Juventa.

Sachße, C., \& Tennstedt, F. (1988). Fürsorge und Wohlfahrtspflege 1871-1929. Geschichte der Armenfürsorge in Deutschland, Bd. 2. Stuttgart: Kohlhammer.

Winkler, M. (2003). Theorie der Sozialpädagogik. Eine Rekonstruktion. Zeitschrift für Sozialpädagogik, 1, 6-23. 